\title{
Attitude Estimation Based on Arrays of Passive RFID Tags
}

\author{
Guillermo Alvarez-Narciandi, Jaime Laviada, Marcos R. Pino, and Fernando Las-Heras, Senior Member, IEEE
}

\begin{abstract}
In this contribution a method to estimate the attitude of an object using uniform arrays of RFID tags is presented. This technique is based on using the MUSIC algorithm with the phase measurements of the signals backscattered by the RFID tags of the array. Two array topologies have been studied (uniform linear arrays (ULA) and uniform circular arrays (UCA)). The performance of the system has been assessed by means of simulations and measurements in a controlled environment and in an indoor scenario. Results show that, after calibration, the attitude can be estimated with an error of a few degrees for a large range of orientations as long as the signals sent by the RFID reader do not imping the tags at grazing angles.
\end{abstract}

Index Terms-RFID; attitude estimation; MUSIC; Uniform Circular Array; Uniform Linear Array.

\section{INTRODUCTION}

$\mathbf{T}$ HE use of Radio Frequency IDdentification (RFID) technology enables optimized tracking and warehouse management resulting in an improvement of logistics efficiency and the subsequent cost savings [1]. The low cost and lack of maintenance of passive RFID tags, which do not require a battery, make them suitable to track pallets or boxes providing identification with non line of sight (NLOS) conditions. RFID readers can be equipped on forklifts or deployed along the supply chain to query the tags identifying the different goods. During this process, the RFID reader sends a signal to a tag attached to a product, which backscatters the signal including its code. Once the reader receives the backscattered signal, the code of the tag, which identifies the product, can be obtained.

In addition to the code of the tag, physical information of the signal such as the received signal strength (RSS) and its phase can be retrieved. During the last years, research effort has been made to develop systems and techniques that exploit this information. In particular, the development of positioning systems has been of great interest. Most of them are based on RSS measurements of the signal backscattered by RFID tags, as this is the parameter provided by most of the commercial readers [2], [3], [4]. However, there are other recent developments based on phase information of the backscattered signal such as [5], [6], [7], [8], [9], where tag positions are estimated using measurements from several antennas, or [10], where the trajectory of the tagged item

This work has been supported by the Gobierno del Principado de Asturias (PCTI)/FEDER-FSE under project GRUPIN14-114; by the Ministerio de Economía y Competitividad of Spain /FEDER under projects TEC2014 55290-JIN and MIRIIEM-TEC2014-54005-P and under the FPU grant FPU15/06431.

Guillermo Alvarez-Narciandi, Jaime Laviada, Marcos R. Pino, and Fernando Las-Heras are with the Departamento de Ingeniería Eléctrica, Universidad de Oviedo, Spain (e-mail: ganarciandi@tsc.uniovi.es; jlaviada@tsc.uniovi.es; mpino@tsc.uniovi.es; flasheras@tsc.uniovi.es). is known and only one antenna is used. In general, these phase-based approaches are developed by resorting to RFID tags working in the UHF bands according to the EPC Gen2 standard [11] and the different regional frequency regulations.

Apart from location estimations, the knowledge of the attitude of goods provides complementary information, which can be very useful to automate logistic processes or when handling with fragile cargo. This information can be obtained with inertial sensors (although they are more expensive than RFID tags and require batteries), using RFID tags and antennas with high directivity as proposed in [12], or by means of a computer vision system (which requires line of sight conditions) [13].

In this paper a novel technique to estimate the attitude of objects based on using an array of RFID tags and a single antenna is presented. The proposed method employs phase measurements of the RFID tags of the array and a direction finding algorithm to estimate the attitude of the array. Some previous work regarding azimuth estimations was presented in [14].

Multiple signal classification (MUSIC) [15] has been selected as the direction finding algorithm, as it has demostrated a good performance for a large variety of situations [16], [17]. This is a high resolution algorithm which is based on the eigendecomposition of the covariance matrix of the input data and requires the knowledge of the array geometry (theoretically or by calibration) to compute the array steering vectors. The steering vectors are the phase shifts between each of the elements of the array and a reference when an incident plane wave impings the array from a given direction.

If two different incident angles result in the same phase distribution along the elements (tags), then an ambiguity in the azimuth and elevation happens. In [18] it was demonstrated that only $3 \mathrm{D}$ arrays can have linearly independent steering vectors for every possible incident direction. A sufficient condition for that linear independence is that the array must have at least 4 non-coplanar elements such that if the first element is placed at the origin of coordinates and each of the rest elements are located in a different reference axis, the distance between the first element and the other 3 must be less than $\lambda / 2$.

In addition, it was demonstrated that in planar arrays, such as a uniform circular array (UCA), only pairs of directions have the same steering vectors if the array has at least 3 noncolinear elements such that if one element is at the origin of coordinates and each of the other two is in a different axis of the array plane and the distance between theese two elements and the first one is less than $\lambda / 2$. Hence, if the previous condition is satisfied, the steering vectors are unique within each of the two subspaces separated by the array plane. Thus, 
it is possible to obtain unambiguously the direction of arrival (DoA) of the signals impinging the planar array restraining the search space to one of those two subspaces.

Finally, in [18] an equivalent condition is also given for 1D DoA estimations using uniform linear arrays (ULA), in which the space between elements is uniform: the element spacing must be less than $\lambda / 2$.

Taking that into account, we will focus in two different flat arrays: 1) uniform linear arrays; 2) uniform circular arrays, in which all the elements of the array are uniformly spaced over a circumference of a given radius. Using the proposed method, unambiguous azimuth, $\alpha$, estimations can be retrieved with an ULA in an angular range of $\left[-90^{\circ}, 90^{\circ}\right]$ and both azimuth and elevation, $\beta$, can be obtained using an UCA for $\alpha \in\left[-90^{\circ}, 90^{\circ}\right]$ and $\beta \in\left[-90^{\circ}, 90^{\circ}\right]$.

The rest of the paper is organized as follows: first, the attitude estimation method is described in Section II. In Section III the numerical results of the simulations carried out to test the system are presented. After that, in Section IV the results of the measurements performed to evaluate the real performance of the system are discussed and finally, the conclusions are drawn.

\section{AtTitude EStimation Method}

The proposed scheme to retrieve the attitude of an object is based on a uniform array of $N$ RFID tags and, at least, one antenna. As previously stated, this work focuses on flat tags arrays. In particular, ULA and UCA topologies are considered enabling to estimate either only azimuth $(\alpha)$ or azimuth and elevation $(\beta)$, respectively. Roll estimation is not considered as it would require the use of a 3D array. An example of this scheme for an ULA of $N=3$ and an UCA of $N=8$ tags is depicted in Fig. 1a and Fig. 1b, respectively.

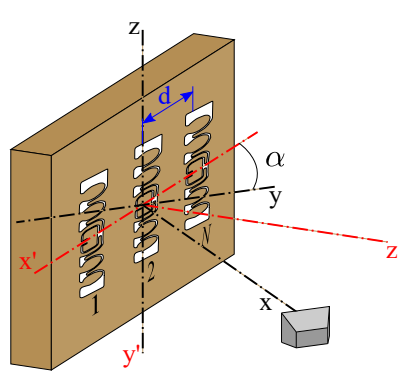

(a)

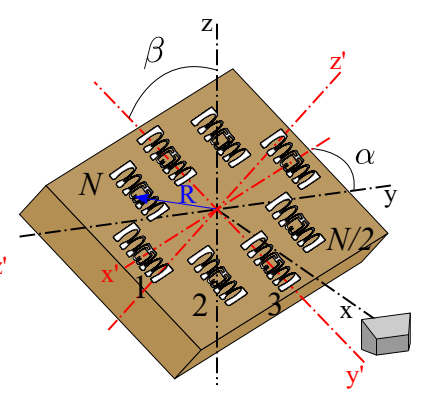

(b)
Figure 1. Scheme of the proposed setup to retrieve the attitude of an object using an ULA of $N=3$ tags (a), and using an UCA of $N=8$ tags (b).

In order to define the attitude estimation, it is necessary to define a global coordinate system (GCS) whose coordinates are denoted as $x, y$ and $z$. Without loss of generality, the center of the array will be considered the origin of the GCS and the transmitter antenna will be placed at the $x$-axis $\left(\vec{r}_{a n t}=\right.$ $\left.\left(x_{a n t}, 0,0\right)\right)$ and pointed towards the origin of the GCS. In addition, an array coordinate system (ACS), denoted by the primed coordinates $x^{\prime}, y^{\prime}, z^{\prime}$, is also defined. The array plane, i.e., where the RFID tags are placed, lies on the $x^{\prime}-y^{\prime}$ plane and the $z^{\prime}$-axis points outwards. Hence, when both the azimuth and elevation are zero $\left(\alpha=0^{\circ}\right.$ and $\left.\beta=0^{\circ}\right)$ the axes of the ACS can be identified with those of the GCS as follows: $x^{\prime}=-y$, $y^{\prime}=-z$ and $z^{\prime}=x$. The ACS can be aligned with the GCS performing one rotation in azimuth in the ULA case and two sequential rotations (first in azimuth and then in elevation) in the UCA case. Therefore, the azimuth angle is the angle between the $y$-axis of the GCS and the - $x$ '-axis of the ACS; in the case of using an UCA, the elevation angle is the angle between the z-axis of the GCS and the -y'-axis of the ACS.

The goal of the attitude estimation is to find the rotation angle or pair of angles, which enables to translate the coordinates from the GCS to the ACS. The attitude estimation method relies on phase measurements of the signals backscattered by the tags, which are used to estimate the direction of arrival of the RFID signals impinging into the array and, afterwards, to compute the aforementioned rotation angles defined in Fig. 1. The direction of arrival of the signals of the RFID reader is given by the angles $\phi$, measured counterclockwise from the $x^{\prime}$-axis in the $x^{\prime}-y^{\prime}$ plane, and $\theta$, which is the polar angle measured from the $z$-axis. As it will be shown later, these angles are univoquely related to the rotation angles $\alpha$ and $\beta$.

In order to estimate the direction of arrival the MUSIC algorithm is used. The general model of the input data, $\mathbf{y}=\left[y_{1} \ldots y_{N}\right]$, for an array of $N$ elements and a single impinging signal is

$$
\left[\begin{array}{c}
y_{1} \\
\vdots \\
y_{N}
\end{array}\right]=\boldsymbol{a}(\theta, \phi) s+\mathbf{n},
$$

where $s$ is the incident signal to the array transmitted by the reader, $\mathbf{n}$ is a vector modeling the noise at each tag and $\boldsymbol{a}(\theta, \phi)$ is the array steering vector of the incident signal, which has direction of arrival $(\theta, \phi)$. The expression of $\boldsymbol{a}(\theta, \phi)$ will be particularized in the next two subsections for the ULA and the UCA case. The direction of arrival of the impinging signals can be obtained from the peak of the MUSIC pseudospectrum, whose expression is

$$
P_{M U S I C}(\theta, \phi)=\frac{1}{\boldsymbol{a}(\theta, \phi)^{H} \boldsymbol{V}_{\boldsymbol{N}} \boldsymbol{V}_{\boldsymbol{N}}^{\boldsymbol{H}} \boldsymbol{a}(\theta, \phi)},
$$

where $V_{N}$ is the matrix which contains the eigenvectors of the noise subspace. In Fig. 2a the pseudospectrum computed for an ULA of $N=5$ tags, $d=\lambda / 8$ and $\alpha=-40^{\circ}$ is depicted whilst in Fig. 2b the pseudospectrum (in 2D) obtained with an UCA of $N=8$ tags, $R=12 \mathrm{~cm}, \beta=-50^{\circ}$ and $\alpha=30^{\circ}$ is shown. As can be seen in Fig. 2, the attitude of an array of passive RFID tags can be inferred from the peak of the pseudospectrum.

Finally, the azimuth and elevation angles can be computed, respectively, from the estimated direction of arrival with the following equations:

$$
\begin{aligned}
& \alpha=\arcsin (\sin (\theta) \cos (\phi)), \\
& \beta=\arctan (\tan (\theta) \sin (\phi)) .
\end{aligned}
$$




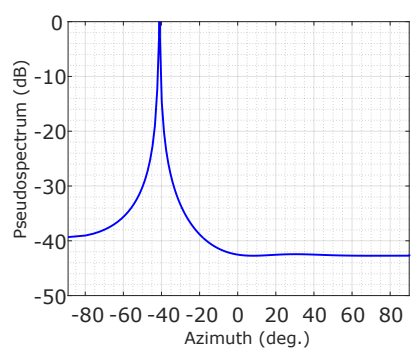

(a)

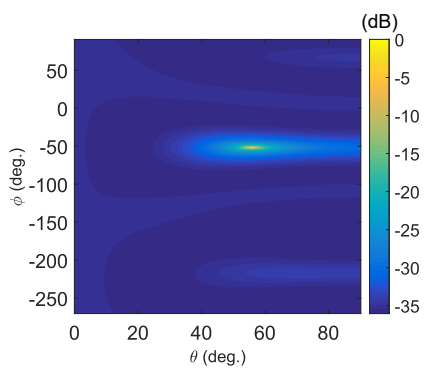

(b)
Figure 2. MUSIC 1D pseudospectrum obtained with an ULA of $N=5$ tags, $d=\lambda / 8$ and $\alpha=-40^{\circ}$, (a), and MUSIC 2D pseudospectrum obtained with an UCA of $N=8$ tags, $R=12 \mathrm{~cm}, \beta=-50^{\circ}$ and $\alpha=30^{\circ}$, (b).

It must be pointed out that the angles are restricted to the ranges $\alpha \in\left[-90^{\circ}, 90^{\circ}\right]$ and $\beta \in\left[-90^{\circ}, 90^{\circ}\right]$ (in the UCA case) and, consequently, the signals never imping the array from the back. Otherwise, an ambiguity would happen and it could only be resolved by resorting to additional arrays or readers. To obtain equations 3 and 4 , first, the expression of the axes of the ACS in terms of the GCS axes, $\alpha$ and $\beta$ was derived:

$$
\begin{aligned}
& x^{\prime}=-\boldsymbol{R}(\alpha, \beta) \cdot y, \\
& y^{\prime}=-\boldsymbol{R}(\alpha, \beta) \cdot z, \\
& z^{\prime}=\boldsymbol{R}(\alpha, \beta) \cdot x,
\end{aligned}
$$

where

$$
\begin{aligned}
\boldsymbol{R}(\alpha, \beta)= & \boldsymbol{R}_{a}(\alpha) \boldsymbol{R}_{e}(\beta)=\left(\begin{array}{ccc}
\cos (\alpha) & -\sin (\alpha) & 0 \\
\sin (\alpha) & \cos (\alpha) & 0 \\
0 & 0 & 1
\end{array}\right) \\
& \left(\begin{array}{ccc}
\cos (\beta) & 0 & \sin (\beta) \\
0 & 1 & 0 \\
-\sin (\beta) & 0 & \cos (\beta)
\end{array}\right),
\end{aligned}
$$

is the rotation matrix to perform two sequential rotations (first in azimuth, $\boldsymbol{R}_{a}(\alpha)$, and then in elevation, $\left.\boldsymbol{R}_{e}(\beta)\right)$. Second, taking into account that the direction of arrival of the signals is always the $x$-axis, the $x$-axis was expressed in terms of the axes of the ACS in the GCS coordinates, $\alpha$ and $\beta$ (Eq. 5, 6 and 7). Then, the coordinates of $x$-axis in the ACS were identified with the direction of arrival in terms of $\theta$ and $\phi$ (Eq. 9, 10 and 11).

$$
\begin{gathered}
\sin (\alpha)=\cos (\phi) \sin (\theta), \\
\cos (\alpha) \sin (\beta)=\sin (\phi) \sin (\theta), \\
\cos (\alpha) \cos (\beta)=\cos (\theta) .
\end{gathered}
$$

\section{A. Uniform Linear Array}

As previously stated, the ULA can only estimate azimuth rotations and, therefore, it is assumed that the array can only rotate along $z$-axis. This is a common situation as when a box or a pallet are stored, they are usually placed upwards but they may be rotated. Therefore, $\beta=0^{\circ}, \phi=0^{\circ}$ and $\alpha=\theta$. Hence, considering far field conditions and taking into account the round-trip of the signals, the phase difference between two consecutive tags is given by

$$
\Delta \varphi=2 \frac{2 \pi}{\lambda} d \sin (\alpha)
$$

where $\lambda$ is the wavelength of the signal and $d$ is the spacing between elements (see Fig. 1a). Consequently, the maximum unambiguous inter element separation is $\lambda / 4$ and the expression of the array steering vectors, considering the first element as reference, can be particularized to

$$
\boldsymbol{a}(\alpha)=\left[\begin{array}{lll}
1 & \ldots & e^{-j \frac{4 \pi}{\lambda} d \sin (\alpha)(N-1)}
\end{array}\right] .
$$

It is important to note that, in contrast to standard MUSIC, a 2 factor has been included in the exponent to account for the round-trip of the backscattered signals [19].

\section{B. Uniform Circular Array}

In the case of an UCA, the array steering vector of the incident signal, which has direction of arrival $(\theta, \phi)$ can be particularized to

$$
\boldsymbol{a}(\theta, \phi)=\left[\begin{array}{c}
e^{-2 j k R \cos \left(\phi-\gamma_{1}\right) \sin (\theta)} \\
\vdots \\
e^{-2 j k R \cos \left(\phi-\gamma_{N}\right) \sin (\theta)}
\end{array}\right],
$$

where $k$ is the wavenumber and $\gamma_{n}$ is the angle of the $n$ th element of the array measured counterclockwise from the $x^{\prime}$-axis, and $R$ is the radius of the array. This expression of the steering vectors corresponds to the traditional equation for a standard angle of arrival problem [20], but again a factor of 2 has been introduced in the exponent due to the round-trip of the backscattered signal. As a consequence, the maximum element spacing given in [18] for planar arrays must be reduced to $\lambda / 4$.

It is relevant to notice that the positions of the elements of the array have origin symmetry. Therefore, if the number of elements is even, the value of $\gamma_{n+N / 2}$ can be expressed in terms of $\gamma_{n}$ as follows [20]:

$$
\gamma_{n+N / 2}=\frac{2 \pi}{N}\left(\frac{N}{2}+n-1\right)=\gamma_{n}+\pi,
$$

and thus, if the phase of the impinging signal at the origin of coordinates is zero, the steering vector of the $n$-th element is the conjugate of the steering vector of the $n+N / 2$-th element:

$$
a_{n}(\theta, \phi)=a_{n+N / 2}^{*}(\theta, \phi)
$$


As a consequence, if an RFID tag of the array is not read but its opposite element is, it would be possible to estimate the phase of the missing backscattered signal:

$$
\angle a_{n}(\theta, \phi)=-\angle a_{n+N / 2}(\theta, \phi) .
$$

This increases the robustness of the proposed system. However, in practice, the read phase does not correspond to a wave with zero phase at the origin of coordinates and, consequently, there is a phase offset, $\gamma$, due to the distance between the antenna and the center of the array, so that the phasor diagram of the backscattered signals is rotated as depicted in Fig. 3.

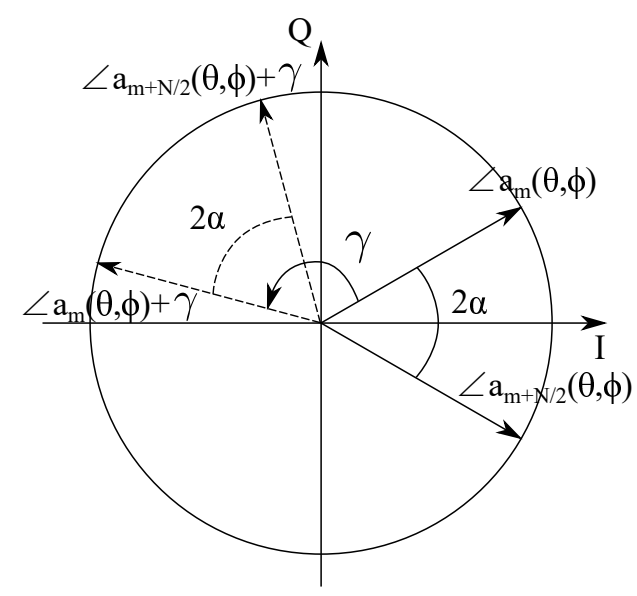

Figure 3. Phasor diagram of the signals backscattered by two opposite RFID tags of the array and its rotated version.

Hence, to enable the estimation of the phase of missing backscattered signals, the value of the phase offset must be computed. This can be done solving the following system of equations as long as, at least, a pair of opposite RFID tags is read. As it can be inferred, the more pairs are read, the more accurate is the estimation of $\gamma$.

$$
\angle a_{n}(\theta, \phi)+\angle a_{n+N / 2}(\theta, \phi)-\gamma=0 \quad n=1 \ldots \frac{N}{2} .
$$

\section{NUMERICAL RESULTS}

In order to evaluate the performance of the proposed method several simulations were conducted. Specifically, the effect of errors in the position of the elements of the array, both the ULA and the UCA, was assesed to test the robustness of the method. Also, the impact of discrepancies between the expected and the actual radius of the UCA is analysed. An analogous analysis was performed for the ULA case taking into account the differences between the expected and the actual element spacing. However, the results of this last study are not reported here as they were presented in [14]. All these simulations, whose results are reported in Sections III-A and III-B, were performed using MatLab software and using a frequency in the European RFID band: $f=866.3 \mathrm{MHz}$. In these simulations, the phase of the received field was set to be proportional to the round-trip distance from the reader to the tag. The obtained phase was introduced in (2) to compute the tilts. In addition, the effect of the couplings and the electromagnetic properties of the surface below the tags was evaluated by means of simulations using the software Feko. The obtained results are discussed in Section III-C.

\section{A. Uniform Linear Array}

The effect of misplacement of tags in an ULA was evaluated through several simulations. The simulated azimuth ranged from $-70^{\circ}$ to $70^{\circ}$ with steps of $10^{\circ}$. For every azimuth angle each simulation was repeated 50 times. Also, the number of elements of the simulated ULA was $N=5$, the element spacing was set to $\lambda / 8$ and the signal to noise ratio (SNR) for the simulations was set to $20 \mathrm{~dB}$. The choice of an element spacing of $\lambda / 8$ instead of the maximum value to avoid unambiguities, $\lambda / 4$, was made to try to thoroughly replicate the measurement setup of Section IV-A, in which the element spacing was reduced to overcome the phase ambiguity introduced by the RFID reader as will be explained. The positioning errors in each direction of the array plane were generated randomly using a uniform distribution defined in the interval $[-a, a]$, where the value of $a$ (the maximum possible value of the error) was modified from 0 (without positioning errors) to $5 \mathrm{~cm}$. The error of the estimated azimuth for each value of $a$ is depicted with blue asterisks in Fig. 4. As it can be seen, for approximately $a<1.5 \mathrm{~cm}$ the error is below $5^{\circ}$ (below the green line).

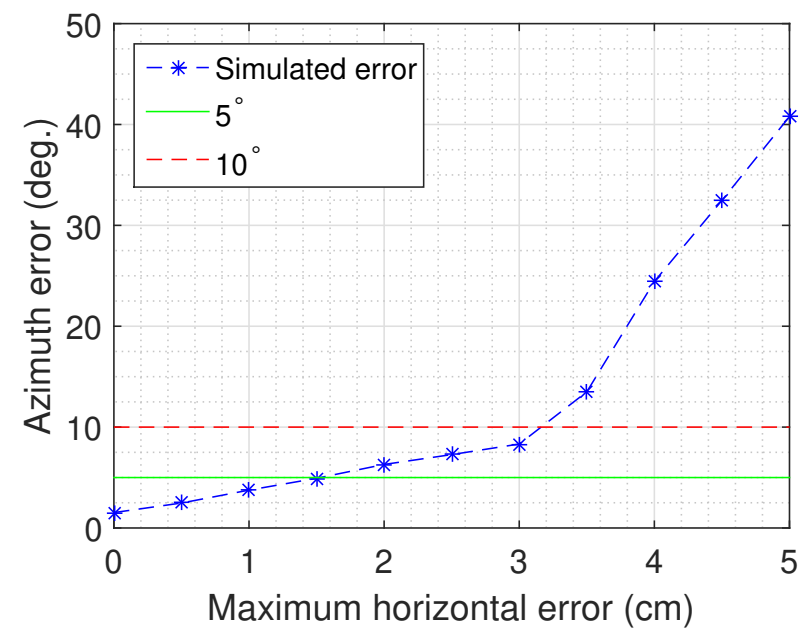

Figure 4. Retrieved azimuth error for $\alpha \in[-70,70]$ with an ULA of $N=5$ for different positioning errors (blue asterisks).

\section{B. Uniform Circular Array}

1) Effect of discrepancies between the theoretical radius and the real one: Several simulations were carried out in order to see how errors on the array radius affect the performance of the system. The obtained results are shown in Fig. 5, where the estimated azimuth and elevation are depicted when: i) the theoretical value of the radius is equal to its real value (blue asterisks); ii) the expected value is bigger than its real value (red circles); and iii) the expected value is smaller than the real one (black "x"). As it can be seen, when the expected 


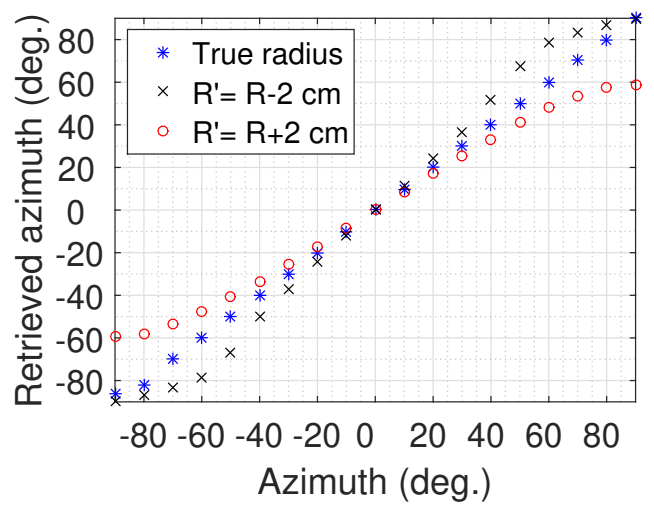

(a)

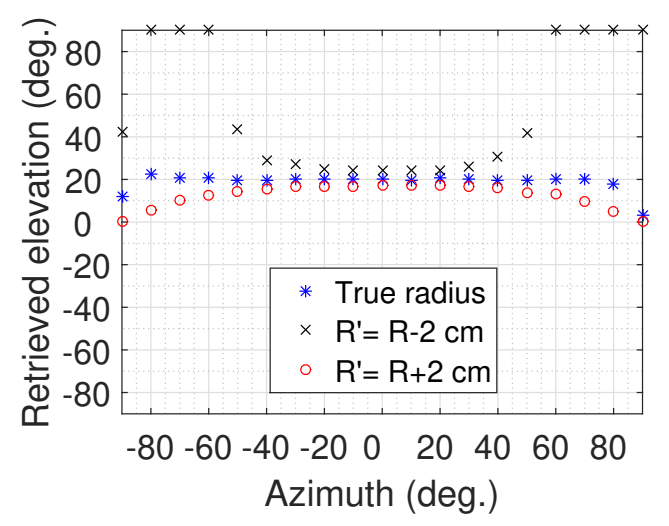

(b)

Figure 5. Retrieved azimuth, (a), and elevation, (b), for $\alpha \in[-90,90]$ with $10^{\circ}$ steps and $\beta=20^{\circ}$ when the expected radius of the UCA was equal to its real value (blue asterisks), smaller than expected (red circles) or greater (black "x").

radius is smaller than the real value the absolute estimated azimuth values are larger than the real ones and the same happens to the estimated elevation values. It should be noted that the difference between estimated and real attitude values are greater as the azimuth of the UCA is increased. Also, the discrepancies between the retrieved elevation values and the real values increase as the true elevation angle is larger. When the expected radius is greater than the real one, as opposed to the previous case, the absolute estimated attitude values are smaller than the real ones. This behaviour is similar to the one reported in [14] for errors in the expected element spacing in ULAs.

2) Effect of positioning errors of the RFID tags: This subsection evaluates the effect on the performance of the system of slightly misplaced RFID tags due to the array manufacturing process. The simulated attitude covered the interval $\left[-70^{\circ}, 70^{\circ}\right]$ in azimuth and $\left[-70^{\circ}, 70^{\circ}\right]$ in elevation with steps of $10^{\circ}$. For every pair of attitude angles, each simulation was repeated 20 times. Also, the number of elements of the simulated UCA was $N=8$ and the signal to noise ratio (SNR) for the simulations was set to $20 \mathrm{~dB}$. As in subsection III-A, the positioning errors in each direction of the array plane were generated randomly using a uniform distribution defined in the interval $[-a, a]$, where the value of $a$ (the maximum possible value of the error) was modified from 0 (without positioning errors) to $5 \mathrm{~cm}$. The error of the estimated azimuth and elevation for each value of $a$ is depicted in Fig. 6a and Fig. $6 \mathrm{~b}$ respectively. The green line encloses the area where the error of the estimated attitude is below $5^{\circ}$ and the red dashed line encloses the area where the error is below $10^{\circ}$. By analysing the results of the simulations, the error in both azimuth (Fig. 6a) and elevation (Fig. 6b) is below $5^{\circ}$ when $a<6 \mathrm{~mm}$.

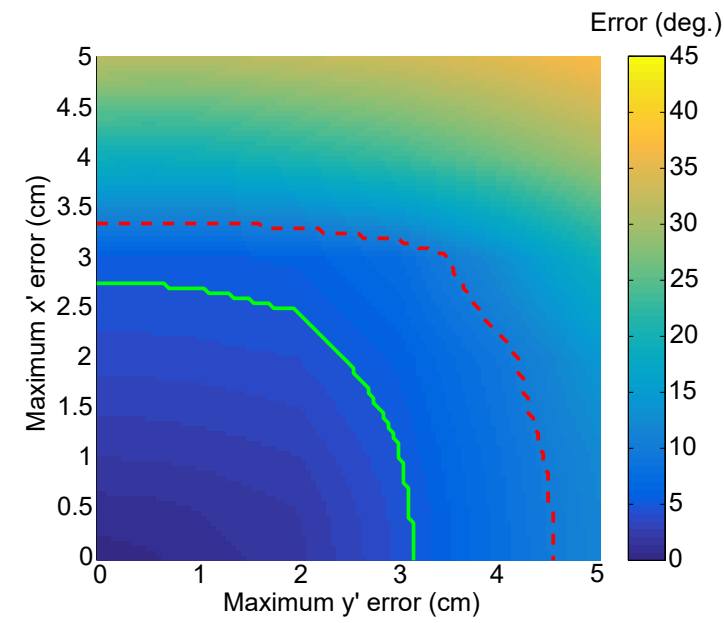

(a)

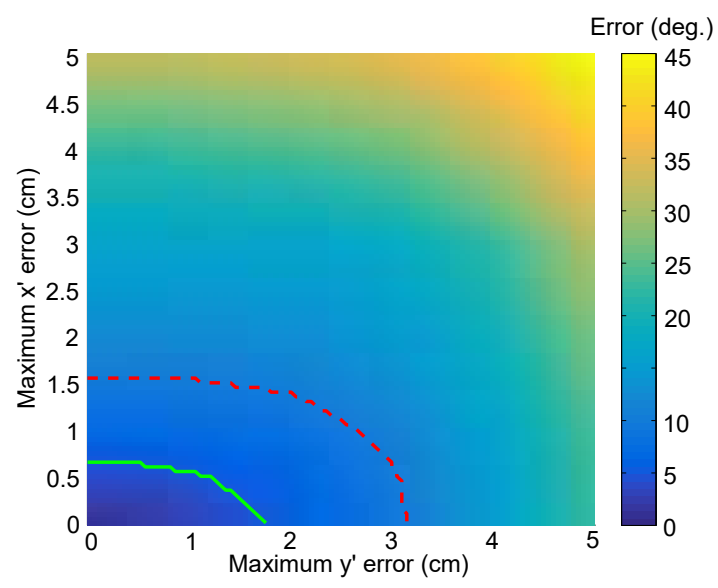

(b)

Figure 6. RMS error of the retrieved azimuth, (a), and the retrieved elevation, (b), for different values of positioning error of the RFID tags of the UCA in both directions of the array plane. The green line encloses the area where the error is below $5^{\circ}$ and the red dashed line encloses the area where the error is below $10^{\circ}$.

\section{Effect of couplings and electromagnetic properties of the material under the tags}

In order to test the effect of couplings between tags an ULA of $N=5$ tags was simulated in Feko for several values of element spacing $d$. The simulated tag model is based on the one proposed in [21]. The simulated azimuth ranged from $-70^{\circ}$ to $70^{\circ}$ and the frequency was set to $f=866.3 \mathrm{MHz}$. To compute the phase of the signals backscattered by a given tag, the activated tag was loaded to the complex conjugate 
impedance of the antenna and the rest of the tags of the array were short-circuited [22].
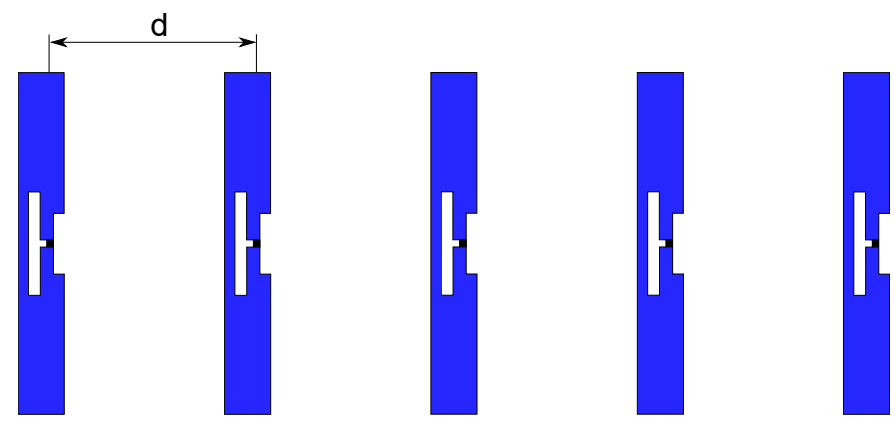

Figure 7. Simulated array of tags.

The obtained results are summarized in Table I, where $\epsilon_{r m s}$ is the root-mean-square (RMS) error of the estimated azimuth, $\sigma$ is the standard deviation, $\Delta$ is the correction term added to the expected value of the element spacing $(d)$, and $\epsilon_{r m s, \Delta}$ and $\sigma_{\Delta}$ are the RMS error and the standard deviation of the estimated azimuth after the correction respectively. As can be seen, as the distance between elements decreases, i.e, there are more couplings between elements, the RMS error increases. However, after the expected inter element spacing is increased by $\Delta$, the effect of couplings between elements is mitigated. In addition, it must be noted that the simulated tag model is very simple and it was not optimized and, thus, the effect of couplings is expected to be lower in the well-designed commercially available RFID tags.

Table I

RMS ERROR AND STANDARD DEVIATION WITH AND WITHOUT CORRECTION OF THE ESTIMATED AZIMUTH WITH AN ULA OF $N=5$ TAGS FOR DIFFERENT ELEMENT SPACING.

\begin{tabular}{|c|c|c|c|c|c|}
\cline { 2 - 6 } \multicolumn{1}{c|}{} & $\epsilon_{r m s}\left({ }^{\circ}\right)$ & $\sigma\left(^{\circ}\right)$ & $\epsilon_{r m s, \Delta}\left({ }^{\circ}\right)$ & $\sigma_{\Delta}\left({ }^{\circ}\right)$ & $\Delta(\mathrm{mm})$ \\
\hline No coupling & 1.3 & 0.6 & - & - & - \\
\hline$d=\lambda / 4$ & 3.9 & 1.9 & 2.0 & 1.5 & 3 \\
\hline$d=3 \lambda / 16$ & 7.5 & 4.4 & 3.5 & 2.2 & 4 \\
\hline$d=5 \mathrm{~cm}$ & 11.0 & 6.0 & 5.1 & 3.5 & 6 \\
\hline$d=\lambda / 8$ & 15.1 & 8.9 & 6.8 & 4.5 & 7 \\
\hline
\end{tabular}

The effect of the electromagnetic properties of the material below the array of RFID tags was also tested by adding a substrate of dielectric material underneath the simulated array. The simulated relative permitivities are not very high as the array of tags would be usually placed above cardboard or plastics with low infill percentage. The results are shown in Table II. As can be seen, the higher the permitivity is, the higher the effect of couplings is, which, again, can be compensated after a calibration process.

Table II

RMS ERROR AND STANDARD DEVIATION WITH AND WITHOUT

CORRECTION OF THE ESTIMATED AZIMUTH WITH AN ULA OF $N=5$ TAGS FOR DIFFERENT PERMITIVITY OF THE MATERIAL BELOW THE ARRAY.

\begin{tabular}{|c|c|c|c|c|c|}
\cline { 2 - 6 } \multicolumn{1}{c|}{} & $\epsilon_{r m s}\left({ }^{\circ}\right)$ & $\sigma\left(^{\circ}\right)$ & $\epsilon_{r m s, \Delta}\left(^{\circ}\right)$ & $\sigma_{\Delta}\left({ }^{\circ}\right)$ & $\Delta(\mathrm{mm})$ \\
\hline$\epsilon_{r}=1$ & 7.5 & 4.4 & 3.5 & 2.2 & 4 \\
\hline$\epsilon_{r}=1.1$ & 8.2 & 4.5 & 4.2 & 2.9 & 4.5 \\
\hline$\epsilon_{r}=1.2$ & 9.1 & 4.8 & 5.1 & 4.0 & 5 \\
\hline$\epsilon_{r}=1.4$ & 11.6 & 6.1 & 6.6 & 5.5 & 6 \\
\hline
\end{tabular}

\section{EXPERIMENTAL RESULTS}

Once the performance of the system was characterized by means of simulations, several measurements were performed: first, in a controlled environment and after that, in an indoor scenario. All the measurements were performed with the commercial reader Speedway Revolution Reader of Impinj [23]. It must be pointed out that this reader randomly introduces an ambiguity of $180^{\circ}$ on its phase measurements, i.e, it is not possible to know if the phase value provided by the reader is the true value or that value plus $180^{\circ}$. The phase jumps were corrected with two different approaches (one for the ULA and one for the UCA) as detailed in the following subsections.

\section{A. ULA}

The proposed system was tested using an ULA of $N=5$ RFID tags inside the facilities of the Area of Signal Theory and Communications of the University of Oviedo. The selected tag model was the Smartrac ShortDipole RFID Paper Tag with the Monza 5 chip [24] and the antenna was the WIRA 30 model of KATHREIN [25]. The setup is depicted in Fig. 8, where the ULA was attached to a cardboard box. The ULA was placed onto a platform to manually perform azimuth rotations. Finally, the ULA and the transmitter antenna were aligned using a cross-line laser.

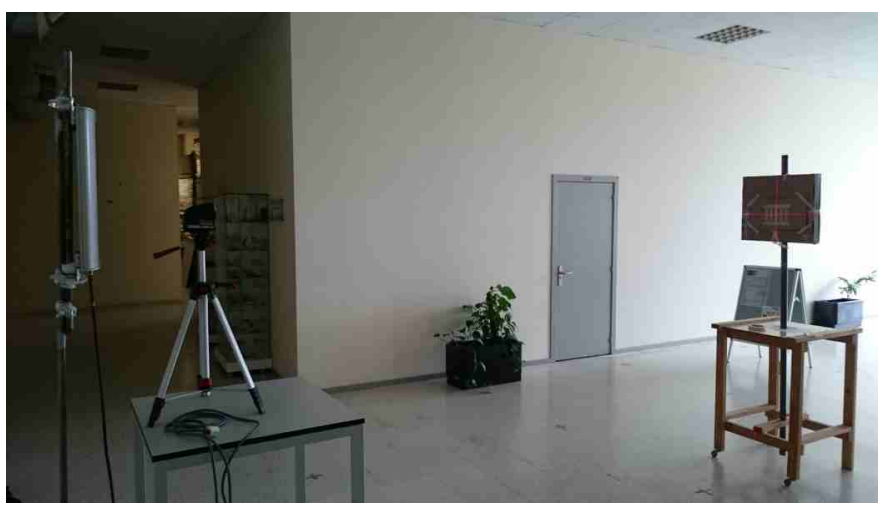

Figure 8. Measurement setup in an indoor environment using an ULA.

The measured azimuth covered from $-90^{\circ}$ to $90^{\circ}$ with $5^{\circ}$ steps. Here, in order to overcome the phase ambiguity introduced by the reader, the element spacing was reduced to $\lambda / 8$ at the expense of higher couplings between the tags. As a consequence, the maximum phase difference between two consecutive tags was reduced to $90^{\circ}$ (see Ec. 12), which made possible to find, without ambiguity, the correct combination of phase values as those which produce the highest and narrowest maximum in the visible region of the MUSIC pseudospectrum. Prior to the tests, a calibration stage with the array at $\alpha=0^{\circ}$ was performed. In this calibration, the offset of each tag of the ULA was measured. Also, a misalignment of the ULA with respect to the rotation axis of $3 \mathrm{~cm}$ in both $x$-axis and $y$-axis was corrected. The obtained results are depicted in Fig. 9 , where the real azimuth values of the ULA are depicted with blue asterisks, the retrieved azimuth values with $d=\lambda / 8$ are shown with black " $x$ " and the retrieved azimuth values, 
where the physical element spacing was reduced to $3.4 \mathrm{~cm}$, are presented with red circles.

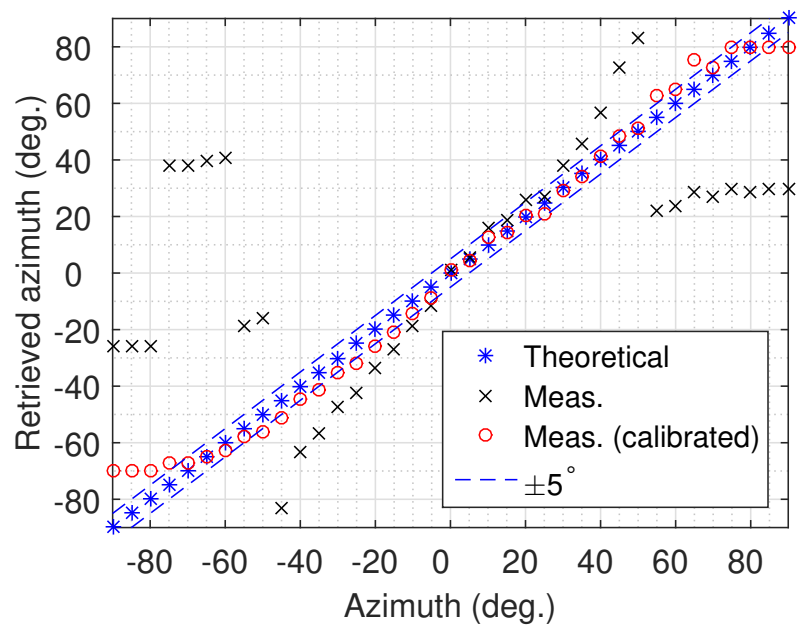

Figure 9. Retrieved azimuth for $\alpha \in[-90,90]$ without calibration (black "x"), with calibration (red circles) and real values (blue asterisks).

As can be seen, the initial results, where the physical element spacing was $\lambda / 8$, show a similar behaviour as the simulations presented in Fig. 2 of [14], where the element separation was greater than expected. Thus, the estimated azimuth is greater than the real values. The greater effective separation between tags than the physical value was due to the electromagnetic properties of the surface below the tags [24], [26]. However, the results after the calibration of the array, where the physical distance between tags was reduced so that the the effective element spacing was $\lambda / 8$, show a good performance of the system in a wide angular range (from $-75^{\circ}$ to $75^{\circ}$ ) with maximum error $\epsilon_{\alpha, \max }=10.6^{\circ}$ and RMS error $\epsilon_{\alpha, r m s}=4.7^{\circ}$.

\section{B. UCA}

The selected RFID tag model for the measurements discussed below was the Smartrac Dogbone [27] with the Monza R6 chip [28] of Impinj and the antenna was the WANTENNAX019 model of CAEN RFID [29]. In this case, the phase ambiguity introduced by the reader was solved by calibrating the system at an initial position and attitude and, then, traking the phase values to correct $180^{\circ}$ increments.

1) Anechoic chamber: The first set of measurements were conducted in the anechoic chamber of the Area of Signal Theory and Communications of the University of Oviedo. The setup is depicted in Fig. 10, were the transmitter antenna was aligned with the center of the UCA by means of a crossline laser. The RFID tags of the UCA were placed on a piece of cardboard which was attached to a $3 \mathrm{D}$ printed plastic support structure. This structure was mounted on a metric goniometer on top of a rotary stage diameter to allow for precise angular adjustment in both azimuth and elevation. The measured attitude ranged from $-90^{\circ}$ to $90^{\circ}$ in azimuth and from $-20^{\circ}$ to $20^{\circ}$ in elevation. The elevation range was limited by the maximum travel of the goniometer. The UCA had
$N=8$ RFID tags and radius of $R=12 \mathrm{~cm}$. The geometry of the UCA was modified in order to minimize couplings between the RFID tags. In particular, the angular separation between RFID tags was not constant in order to increase the distance between tags in the $x^{\prime}$-axis.

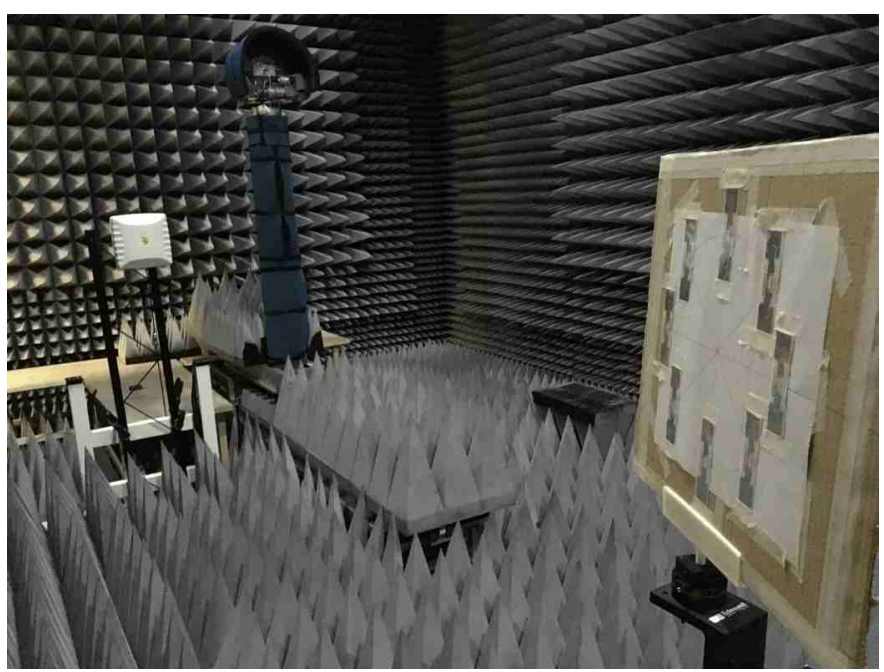

Figure 10. Measurement setup in the anechoic chamber of the University of Oviedo.

Prior to the measurements, a calibration of the UCA was performed for two reasons: first, to compensate the different offset values of the RFID tags; second, to mitigate the effect of small positioning errors of each of the tags of the array, couplings between tags, and misalignments of the UCA and the transmitter antenna. The calibration, formalized in Eq. 19, consisted of matching the theoretical phase values for each RFID tag to the measured ones for the different attitude values by making small changes in their positions, in their offset and in the value of the radius of the UCA (Fig. 11).

$$
\underset{\substack{\overrightarrow{\boldsymbol{r}}_{t a g, 1}, \ldots, \boldsymbol{r}_{t a g, N} \\ \xi_{1}, \ldots, \xi_{N}, R}}{\arg \min }\left|\varphi_{t h}\left(\overrightarrow{\boldsymbol{r}}_{t a g, 1}, \ldots, \overrightarrow{\boldsymbol{r}}_{t a g, N}, \xi_{1}, \ldots, \xi_{N}, R\right)-\varphi_{m e a s}\right|
$$

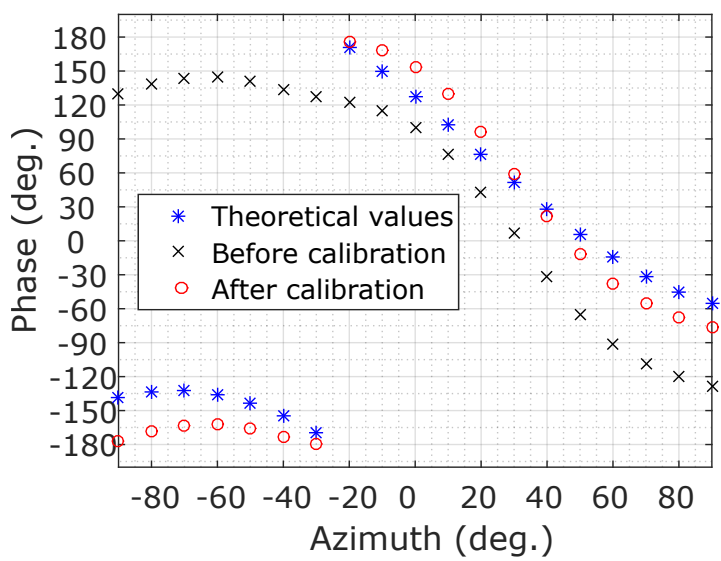

Figure 11. Measured phase of one of the RFID tags of the UCA for $\alpha \in$ $[-90,90]$ and $\beta=-10^{\circ}$ before and afer calibrating and theoretical values. 
The correction terms obtained after the calibration are shown in Table III. Also, the radius was increased $25 \mathrm{~mm}$ to compensate the effect described in Section III-B1.

Table III

CORRECTIONS ON THE POSITION OF THE RFID TAGS OF THE UCA OF THE SETUP OF THE ANECHOIC CHAMBER.

\begin{tabular}{|c|c|c|c|c|c|c|c|c|}
\hline Tag \# & 1 & 2 & 3 & 4 & 5 & 6 & 7 & 8 \\
\hline$z_{\text {corr }}(\mathrm{cm})$ & 18 & 18 & 18 & 18 & 18 & 18 & 18 & 18 \\
\hline$y_{\text {corr }}(\mathrm{mm})$ & 8 & -2 & -2 & 2 & 6 & 2 & 0 & -2 \\
\hline Offset (deg.) & -10 & -30 & -5 & -49 & -24 & -11 & -3 & 1 \\
\hline
\end{tabular}

In Fig. 12 the retrieved azimuth and elevation and the expected values for $\alpha \in[-90,90]$ with $10^{\circ}$ steps and $\beta=10^{\circ}$ for two different expected radius values are depicted.

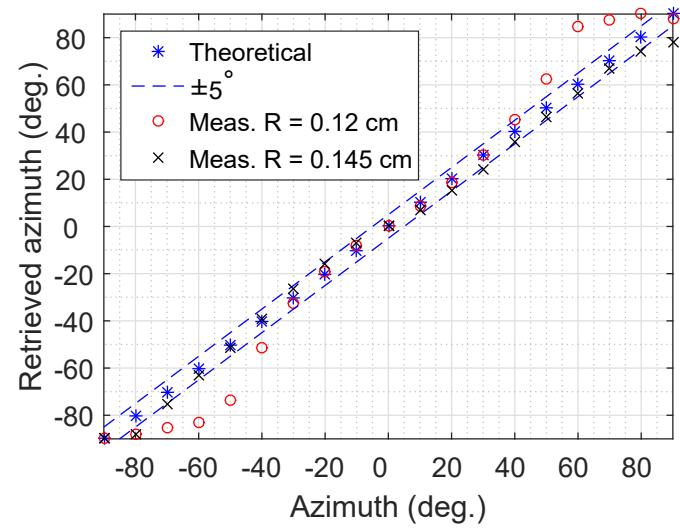

(a)

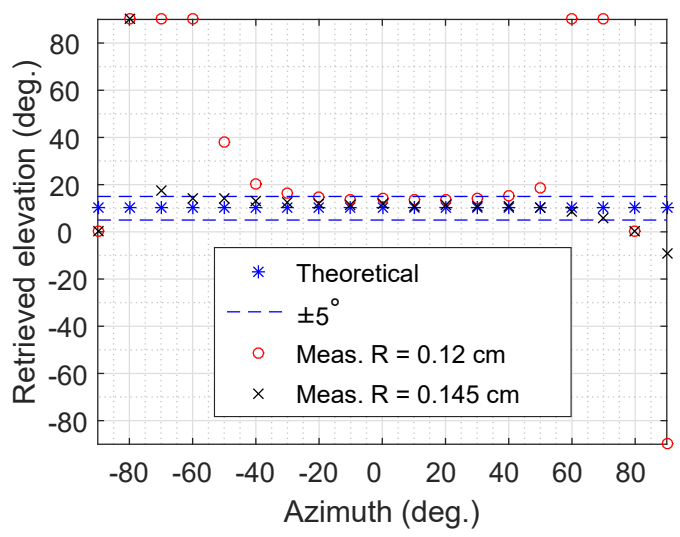

(b)

Figure 12. Retrieved azimuth, (a), and elevation, (b), for $\alpha \in[-90,90]$ with $10^{\circ}$ steps and $\beta=10^{\circ}$ for $R=12 \mathrm{~cm}$ (red circles) ans for $R=14.5 \mathrm{~cm}$ (black "x").

As can be seen, when the expected radius value is increased, the retrieved attitude matches its real value. The difference between the theoretical positions of the UCA elements and the corrected ones are not only due to positioning errors, but also to the electromagnetic properties of the surface below the tags and couplings between them. This effect makes the effective separation between tags larger than their physical value [27], [26]. It must be pointed out that the correction on the $z$-axis, $z_{\text {corr }}$, accounts for the vertical displacement of the center of the UCA with respect to the rotation center of the goniometer (the rotation center of the goniometer is only $4.7 \mathrm{~cm}$ above its scale and, hence, there are not enough space to place the center of the UCA on the rotation center). The corrections on the $y$-axis, $y_{\text {corr }}$, represent errors on the horizontal positions of the tags.

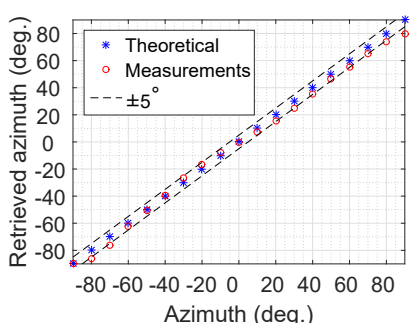

(a)

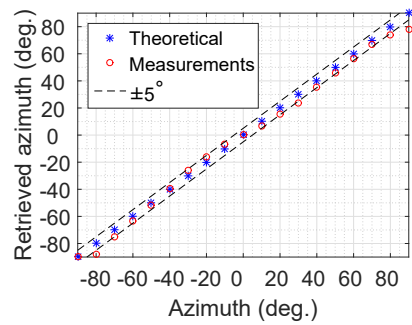

(c)

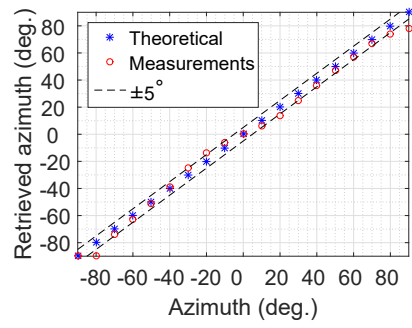

(e)

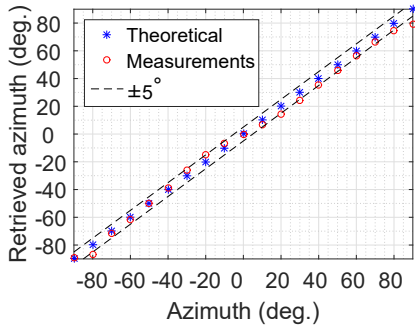

(g)

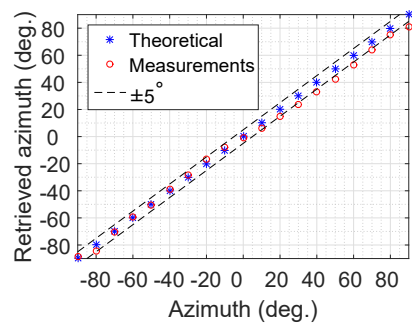

(i)

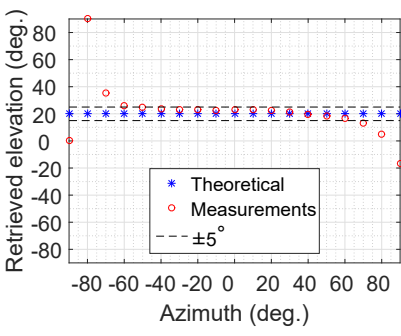

(b)

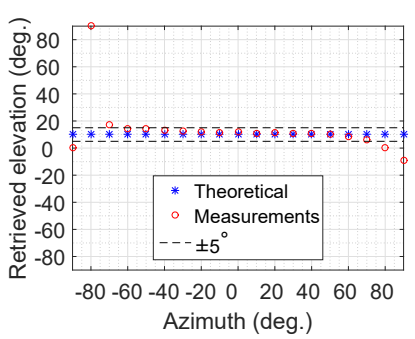

(d)

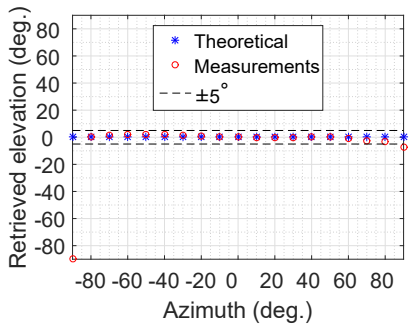

(f)

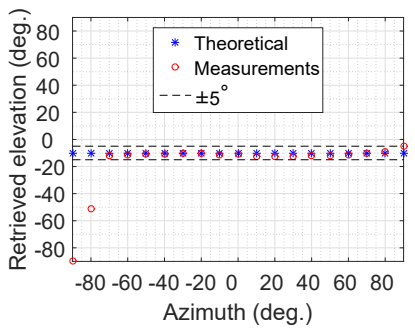

(h)

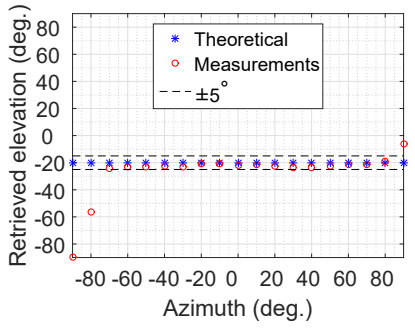

(j)
Figure 13. Retrieved azimuth and elevation for $\alpha \in[-90,90]$ with $10^{\circ}$ steps and $\beta=20^{\circ}$ (a, b); $\beta=10^{\circ}$ (c, d); $\beta=0^{\circ}$ (e, f); $\beta=-10^{\circ}(\mathrm{g}, \mathrm{h})$; and $\beta=-20^{\circ}$, (i, j). 
The obtained results after the calibration are depicted in Fig. 13 for 5 cuts in elevation (from $-20^{\circ}$ to $20^{\circ}$ in $10^{\circ}$ steps) and azimuth ranging from $-90^{\circ}$ to 90 in $10^{\circ}$ steps. As can be seen, the estimated azimuth values are very accurate even for grazing angles. The error of the retrieved elevation is also low, but in this case the accuracy is degraded for grazing angles. This fact is coherent with the higher error sensitivity for the elevation estimation already observed when analyzing the impact of innacuracies in tag positions in azimuth and elevation estimations as shown in Fig. 6a and Fig. 6b, respectively. The maximum and RMS error, $\epsilon_{\max }$ and $\epsilon_{r m s}$ respectively, for both azimuth and elevation for $|\alpha|<60^{\circ}$ and $|\alpha|<70^{\circ}$ are shown in Table IV. Results show that the system provides an accurate estimation of the attitude of the UCA for a wide angular range in azimuth, $\left[-60^{\circ}, 60^{\circ}\right]$, and for all the elevation values that was possible to measure, $\left[-20^{\circ}, 20^{\circ}\right]$.

Table IV

MAXIMUM AND RMS ERROR FOR AZIMUTH AND ELEVATION OF THE ATTITUDE ESTIMATED IN THE ANECHOIC CHAMBER.

\begin{tabular}{|c|c|c|c|c|}
\cline { 2 - 5 } \multicolumn{1}{c|}{} & $\epsilon_{\alpha, \max }\left({ }^{\circ}\right)$ & $\epsilon_{\alpha, r m s}\left({ }^{\circ}\right)$ & $\epsilon_{\beta, \max }\left({ }^{\circ}\right)$ & $\epsilon_{\beta, r m s}\left({ }^{\circ}\right)$ \\
\hline$|\alpha|<60$ & 7.5 & 3.8 & 5.8 & 2.3 \\
\hline$|\alpha|<70$ & 7.5 & 3.9 & 15.6 & 3.1 \\
\hline
\end{tabular}

2) Indoor environment: Once the presented system was validated in a controlled environment, its performance was tested in an indoor scenario. The setup, in a corridor in the research facility of the Area of Signal Theory and Communications of the University of Oviedo, is displayed in Fig. 14. As in the setup presented in section IV-B1, the UCA and the transmitter antenna were aligned by means of a cross-line laser. However, in this case the RFID tags of the UCA were placed on a 3D printed plastic plate instead of on a piece of cardboard. The rest of the structure, built to carefully modify the attitude of the UCA, remained the same. During these tests, it was decided to include a reference RFID tag in the center of the UCA to obtain more information without increasing the size of the array. The array was recalibrated as in Section IV-B1 and the obtained values are shown in Table V.

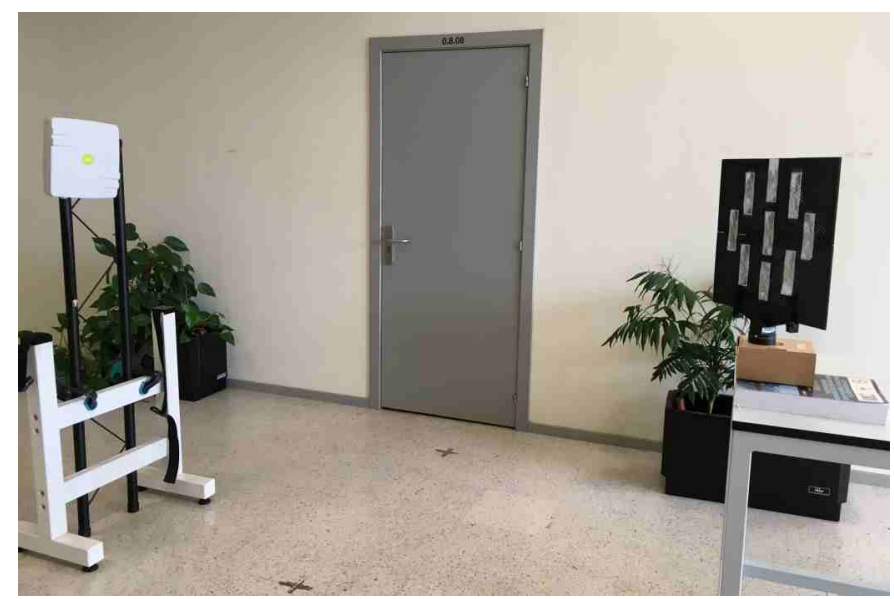

Figure 14. Indoor measurement setup.

The obtained results, discarding the phase measurements of the central tag $(N=8)$ and using all the available data
$(N=9)$, are shown in Fig. 15 for 5 cuts in elevation (from $-20^{\circ}$ to $20^{\circ}$ in $10^{\circ}$ steps) and azimuth ranging from $-90^{\circ}$ to $90^{\circ}$ in $10^{\circ}$ steps.

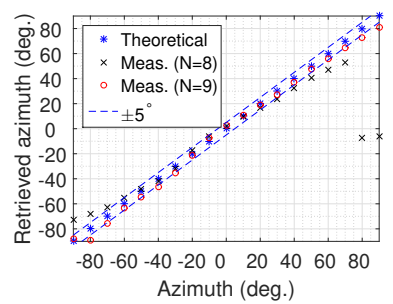

(a)

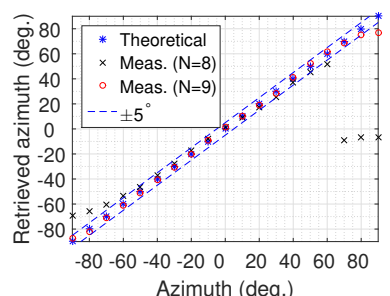

(c)

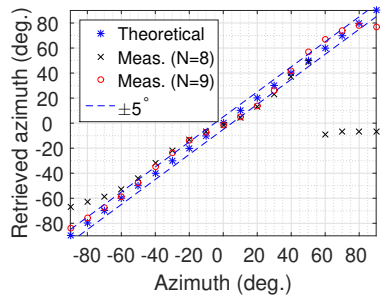

(e)

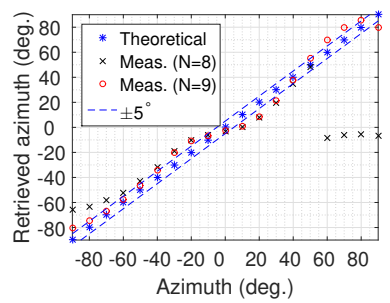

(g)

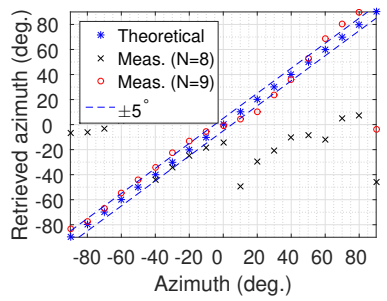

(i)

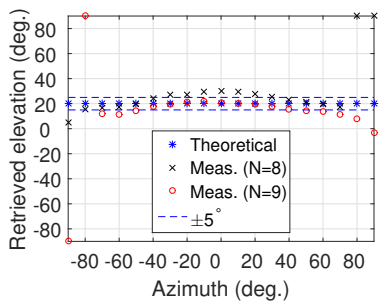

(b)

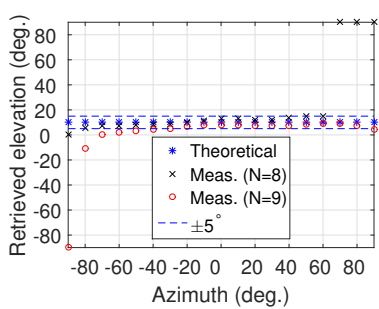

(d)

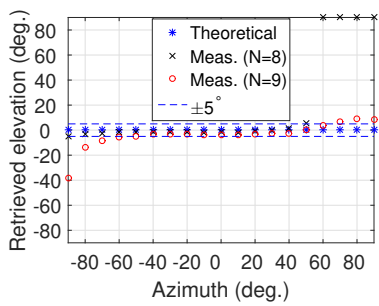

(f)

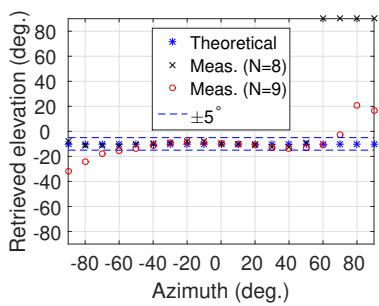

(h)

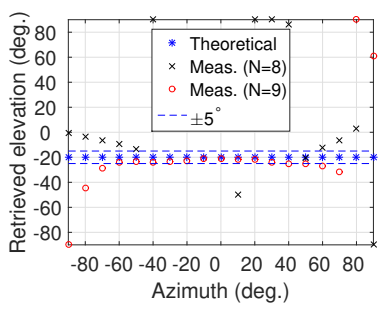

(j)
Figure 15. Retrieved azimuth and elevation for $\alpha \in[-90,90]$ with $10^{\circ}$ steps and $\beta=20^{\circ}$ (a, b); $\beta=10^{\circ}$ (c, d); $\beta=0^{\circ}$ (e, f); $\beta=-10^{\circ}(\mathrm{g}, \mathrm{h})$; and $\beta=-20^{\circ}$, (i, j).

As can be seen, although the performance using $N=8$ tags is good or acceptable for wide attitude range, there are some 
Table V

CORRECTIONS ON THE POSITION OF THE RFID TAGS OF THE UCA OF THE SETUP OF THE INDOOR SCENARIO.

\begin{tabular}{|c|c|c|c|c|c|c|c|c|}
\hline Tag \# & 1 & 2 & 3 & 4 & 5 & 6 & 7 & 8 \\
\hline$z_{\text {corr }}(\mathrm{cm})$ & 21 & 21 & 15 & 15 & 20.4 & 21 & 21 & 21 \\
\hline$y_{\text {corr }}(\mathrm{mm})$ & 23 & 37 & 6 & 33 & -8 & -4 & 8 & 16 \\
\hline Offset $($ deg. $)$ & 55 & 43 & 27 & 62 & 43 & 66 & 77 & 58 \\
\hline
\end{tabular}

values, specially for $\beta=-20^{\circ}$, for which the results are very poor. The error values are summarized in Table VI. However, due to the number of outliers in the azimuth estimations, the median error $\left(6.0^{\circ}\right.$ for $|\alpha|<60^{\circ}$ and $\left.7.0^{\circ}|\alpha|<70^{\circ}\right)$ and the third quartil $\left(9.5^{\circ}\right.$ for $|\alpha|<60^{\circ}$ and $\left.11.7^{\circ}|\alpha|<70^{\circ}\right)$ are more representative than the RMS error this time. As depicted in Fig. 15, the accuracy of the proposed system using a central RFID tag is significantly improved, specially in elevation estimations, as shown in Table VII, where the error values are summarized. The error values are below $10^{\circ}$ in a wide range of attitude values and without increasing the size of the array.

Table VI

MAXIMUM AND RMS ERROR FOR AZIMUTH AND ELEVATION OF THE ATTITUDE ESTIMATED IN THE INDOOR SCENARIO WITH $N=8$.

\begin{tabular}{|c|c|c|c|c|}
\cline { 2 - 5 } \multicolumn{1}{c|}{} & $\epsilon_{\alpha, \max }\left({ }^{\circ}\right)$ & $\epsilon_{\alpha, r m s}\left({ }^{\circ}\right)$ & $\epsilon_{\beta, \max }\left({ }^{\circ}\right)$ & $\epsilon_{\beta, r m s}\left({ }^{\circ}\right)$ \\
\hline$|\alpha|<60$ & 71.7 & 24.5 & 110.0 & 36.8 \\
\hline$|\alpha|<70$ & 79.0 & 29.8 & 110.0 & 38.8 \\
\hline
\end{tabular}

Table VII

MAXIMUM AND RMS ERROR FOR AZIMUTH AND ELEVATION OF THE ATTITUDE ESTIMATED IN THE INDOOR SCENARIO WITH $N=9$.

\begin{tabular}{|c|c|c|c|c|}
\cline { 2 - 5 } \multicolumn{1}{c|}{} & $\epsilon_{\alpha, \max }\left({ }^{\circ}\right)$ & $\epsilon_{\alpha, r m s}\left({ }^{\circ}\right)$ & $\epsilon_{\beta, \max }\left({ }^{\circ}\right)$ & $\epsilon_{\beta, r m s}\left({ }^{\circ}\right)$ \\
\hline$|\alpha|<60$ & 9.9 & 5.0 & 8.9 & 3.7 \\
\hline$|\alpha|<70$ & 11.2 & 5.1 & 11.8 & 4.6 \\
\hline
\end{tabular}

\section{CONCLUSIONS}

In this contribution a novel method to compute the attitude of objects based on phase measurements of RFID passive tags of uniform arrays is presented. Two array topologies were studied: ULAs and UCAs. With the former, azimuth estimations can be obtained whereas with the latter, both azimuth and elevation can be retrieved. In order to evaluate the performance of the proposed method several simulations and measurements were carried out.

The simulations focused on how positioning errors of each of the elements of the array and discrepancies between the expected dimensions of the array and the real ones affect the performance of the system. In the ULA case it was shown that the accuracy of the system was below $5^{\circ}$ for uniformly distributed errors up to $1.5 \mathrm{~cm}$. In the UCA case it was shown that both azimuth and elevation errors were below $5^{\circ}$ for uniformly distributed errors up to $0.6 \mathrm{~cm}$, being the estimated elevation more sensitive to positioning errors than the retrieved azimuth. In addition, it was shown that having an UCA radius greater than expected results in greater retrieved azimuth and elevation than the real attitude of the array.
The system with an ULA of $N=5$ RFID tags was tested in an indoor environment. The obtained results show a good performance with an RMS error below $5^{\circ}$ for $\alpha \in\left[-75^{\circ}, 75^{\circ}\right]$ after the array calibration. For greater azimuth values the accuracy of the system decreases, although in those cases the signals imping the array at grazing angles.

In addition, the proposed method was tested for 2D attitude retrieval with an UCA of $N=8$ tags and also with a reference tag in the center of the array. Results show a good performance of the system in both an anechoic chamber and an indoor scenario. As in the case of the ULA, it should be remarked that the calibration is critical to ensure a good accuracy on the attitude estimations. Concerning this, the corrections regarding couplings between tags can be done in advance taking into account the selected tag models. Thus, it would only be necessary to measure the offset of the given tags of the array before deploying it. The use of a reference tag improves the performance of the system achieving an RMS error close to $5^{\circ}$ for azimuth estimations and below $5^{\circ}$ for the retrieved elevation in a range of $\beta \in\left[-20^{\circ}, 20^{\circ}\right]$ and $\alpha \in\left[-70^{\circ}, 70^{\circ}\right]$.

\section{REFERENCES}

[1] K. Michael and L. McCathie, "The pros and cons of RFID in supply chain management," in Int. Conf. Mobile Business (ICMB'05), July 2005, pp. 623-629.

[2] X. Chen, L. Xie, C. Wang, and S. Lu, "Adaptive accurate indoorlocalization using passive RFID," in Proc. IEEE Int. Conf. Parallel Distrib. Syst. (ICPADS), 2013, pp. 249-256.

[3] S. Shao and R. J. Burkholder, "Item-level RFID tag location sensing utilizing reader antenna spatial diversity," IEEE Sensors J., vol. 13, no. 10, pp. 3767-3774, Oct. 2013.

[4] Y. Alvarez, M. E. D. Cos, and F. Las-Heras, "A received signal strength RFID-based indoor location system," Sens. Actuators A Phys., vol. 255, pp. 118-133, mar 2017.

[5] P. V. Nikitin, R. Martinez, S. Ramamurthy, H. Leland, G. Spiess, and K. V. S. Rao, "Phase based spatial identification of UHF RFID tags," in 2010 IEEE Int. Conf. on RFID, 2010, pp. 102-109.

[6] S. Azzouzi, M. Cremer, U. Dettmar, T. Knie, and R. Kronberger, "Improved aoa based localization of UHF RFID tags using spatial diversity," in Proc. IEEE Int. Conf. RFID-Technol. Appl. (RFID-TA), 2011, pp. 174-180.

[7] M. Scherhäufl, M. Pichler, and A. Stelzer, "UHF RFID localization based on evaluation of backscattered tag signals," IEEE Trans. on Instrum. Meas., vol. 64, no. 11, pp. 2889-2899, Nov. 2015.

[8] J. Huiting, H. Flisijn, A. B. J. Kokkeler, and G. J. M. Smit, "Exploiting phase measurements of EPC Gen2 RFID tags," in Proc. IEEE Int. Conf. RFID-Technol. Appl. (RFID-TA), 2013, pp. 1-6.

[9] M. Scherhäufl, M. Pichler, and A. Stelzer, "Robust localization of passive UHF RFID tag arrays based on phase-difference-of-arrival evaluation," in 2015 IEEE Topical Conf. Wireless Sensors and Sensor Netw. (WiSNet), Jan 2015, pp. 47-49.

[10] A. Buffi and P. Nepa, "The SARFID technique for discriminating tagged items moving through a UHF-RFID gate," IEEE Sensors Journal, vol. 17, no. 9, pp. 2863-2870, may 2017.

[11] GS1. (2016, 11) EPC radio-frequency identity protocols generation-2 UHF RFID. [Online]. Available: http://www.gs1.org/sites/default/files/docs/epc/Gen2_Protocol_Standard. pdf

[12] F. Inui, "RF tag, RF tag attitude detection apparatus, and RF tag attitude detection system," U.S. Patent 20050225 451, Apr. 7, 2004.

[13] C. Rennie, R. Shome, K. E. Bekris, and A. F. D. Souza, "A dataset for improved rgbd-based object detection and pose estimation for warehouse pick-and-place," IEEE Robot. and Autom. Lett., vol. 1, no. 2, pp. 1179 1185, July 2016.

[14] G. A. Narciandi, J. Laviada, and F. Las-Heras, "Object attitude estimation using passive RFID tag arrays," in 2016 URSI Int. Symp. Electromagn. Theory (EMTS), Aug 2016, pp. 572-574.

[15] R. O. Schmidt, "Multiple emitter location and signal parameter estimation," IEEE Trans. Antennas Propag., vol. 34, no. 3, pp. 276-280, Mar. 1986. 
[16] Z. Ye and C. Liu, "2-D DOA estimation in the presence of mutual coupling," IEEE Trans. Antennas Propag., vol. 56, no. 10, pp. 31503158 , Oct 2008

[17] A. Ferreol, P. Larzabal, and M. Viberg, "On the asymptotic performance analysis of subspace DOA estimation in the presence of modeling errors: case of MUSIC," IEEE Trans. Signal Process., vol. 54, no. 3, pp. 907920, March 2006.

[18] L. C. Godara and A. Cantoni, "Uniqueness and linear independence of steering vectors in array space," J. Acoust. Soc. Am., vol. 70, pp. 467475,1981

[19] G. A. Narciandi, J. Laviada, M. R. Pino, and F. Las-Heras, "Application of MUSIC algorithm to the estimation of objects attitude," in Int. Conf. Electromagn. Adv. Appl. (ICEAA), 2017.

[20] Y. Wu and H. C. So, "Simple and accurate two-dimensional angle estimation for a single source with uniform circular array," IEEE Antennas Wireless Propag. Lett., vol. 7, pp. 78-80, 2008.

[21] Y. Ziyuan, H. Xiaoxiang, Y. Yang, and T. Liu, "UHF RFID tag antenna performance on various dielectric-background," in 2016 IEEE International Workshop on Electromagnetics: Applications and Student Innovation Competition (iWEM), May 2016, pp. 1-3.

[22] P. V. Nikitin and K. V. S. Rao, "Theory and measurement of backscattering from rfid tags," IEEE Antennas and Propagation Magazine, vol. 48, no. 6, pp. 212-218, Dec 2006.

[23] Imping. (2016, 10) Imping low level user data. [Online]. Available: https://support.impinj.com/hc/en-us/articles/202755318ApplicationNote-Low-Level-User-Data-Support

[24] Smartrac Technology Gmbh. (2016, 10) Smartrack shortdipole RFID paper tag with Monza 5 chip. [Online]. Available: https://www.smartracgroup.com/files/content/Products_Services/PDF/0018_SMARTRAC SHORTDIPOLE.pdf

[25] KATHREIN Solutions Gmbh. (2017, 8) Wira 30 antenna unit. [Online]. Available: https://www.kathreinsolutions.com/files/52010086_52010087_wira_30_93636225c.pdf

[26] M. Kanesan, D. V. Thiel, and S. G. O'Keefe, "The effect of lossy dielectric objects on a UHF RFID meander line antenna," in Proc. 2012 IEEE Int. Symp. Antennas and Propag., July 2012, pp. 1-2.

[27] Smartrac Technology Gmbh. (2017, 6) Smartrac dogbone monza r6 rfid tag. [Online]. Available: https://www.smartracgroup.com/files/content/Products_Services/PDF/0028_SMARTRAC_ DOGBONE.pdf

[28] Impinj. (2017, 6) Chip monza r6. [Online]. Available: https://support.impinj.com/hc/en-us/articles/202765328-MonzaR6-Product-Datasheet

[29] CAEN RFID. (2017, 8) Wantennax019 antenna. [Online]. Available: https://www.caenrfid.com/en/products/wantennax019/

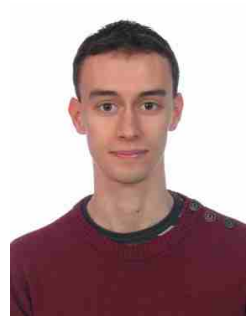

Guillermo Alvarez-Narciandi Guillermo AlvarezNarciandi received the M.Sc. degree in Telecommunication Engineering from the University of Oviedo, Gijón, Spain, in 2016, where he is currently working toward the Ph.D. degree (with the support of a FPU grant from the Spanish Government). He was a Visiting Student at Stanford University, CA, USA, in 2014. He received the Special Award to the Best Entrepreneurship Initiative in the XV Arquímedes national contest in 2017 for the development of a RFID-based location system. He received the 2015 EPIGIJON Industrial Partners Society Award to the Best Applied Project for the development and testing of a hybrid RSS-inertial Indoor Positioning System. He has co-authored a patent on attitude estimation using RFID technology. His main research interests are location systems, attitude estimation systems, RFID technology and imaging techniques.

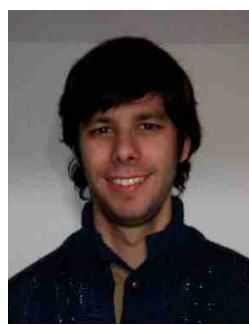

Jaime Laviada Jaime Laviada was born in $\mathrm{Gi}$ jón, Spain. He received the M.S. degree in telecommunication engineering and the Ph.D. degree from the Universidad de Oviedo, Spain (http://www.uniovi.es), in 2005 and 2010, respectively. In 2006, he joined the research group Signal Theory and Communications of the Universidad de Oviedo, where he has been involved in multiple national and European projects as well as contracts with several companies. In 2015, he moved to the Antennas Group of the Universidad Pública de Navarra with a national postdoctoral fellowship collaborating in several applied research projects. Finally, he moved back to the Universidad de Oviedo where he currently holds a position as assistant professor. In addition, he has been a Visiting Scholar in the Electromagnetics and Communications Lab, Pennsylvania State University, during 2007 and 2008 as well as in the Applied Microwave NonDestructive Testing Laboratory, Missouri S\&T, during 2017.

His research interests include numerical techniques applied to electromagnetic imaging, antenna measurements, method of moments, and antenna pattern synthesis.

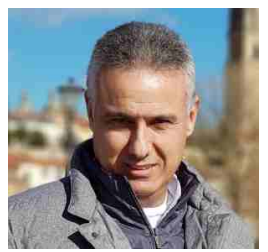

Marcos R. Pino Marcos R. Pino was born in Vigo, Spain, in 1972. He received the M.Sc. and Ph.D. degrees in telecommunication engineering from the University of Vigo, Spain, in 1997 and 2000, respectively.

In 1998, he was a Visiting Scholar at the ElectroScience Laboratory, The Ohio-State University, Columbus, OH, USA. From 2000 to 2001, he was with the University of Vigo as Assistant Professor. Since 2001, he has been with the Electrical Engineering Department, University of Oviedo, Spain, where he is currently Associate Professor teaching courses on communication systems and antenna design.

His research interests include antenna design for different applications (RFID, satellite broadcasting, ... ), both near-field and far-field measurement techniques and facilities, efficient computational techniques applied to solve EM problems, such as evaluation of radar cross section or EM scattering from targets in the presence of rough surfaces.

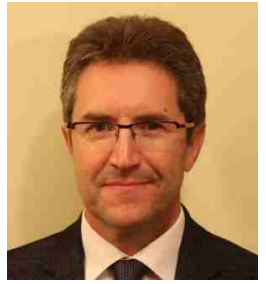

Fernando Las-Heras Fernando Las-Heras (M'86, SM'08) received the M.S. in 1987 and the Ph.D. in 1990, both in Telecommunication Engineering, from the Technical University of Madrid (UPM). He was a National Graduate Research Fellow (19881990) and he held a position of Associate Professor at the Department of Signal, Systems and Radiocommunications of the UPM (1991-2000). From December 2003 he holds a Full-Professor position at the University of Oviedo where he was the Vice-dean for Telecommunication Engineering at the Technical School of Engineering at Gijón (2004-2008). As of 2001 he heads the research group Signal Theory and Communications TSC-UNIOVI at the Dept. of Electrical Engineering of the University of Oviedo. He was a Visiting Lecturer at the National University of Engineering in Peru in 1996, a Visiting Researcher at Syracuse University, New York, in 2000, and a short term Visiting Lecturer at ESIGELEC in France from 2005 to 2011. He held the Telefónica Chair on "RF Technologies", "ICTs applied to Environment" and "ICTs and Smartcities" at the University of Oviedo (2005-2015). Member of the board of directors of the IEEE Spain Section (2012-2015), member of the board IEEE Microwaves \& Antennas Propagation Chapter (AP03/MTT17) (2016-2017), member of the Science, Technology and Innovation Council of Asturias (2010), and president of the professional association of Telecommunication Engineers at Asturias. He has led and participated in a great number of research projects and has authored over 190 technical journal papers, mainly in the areas of antennas, propagation, metamaterials and inverse problems with application to antenna measurement (NF-FF, diagnostics and holography), electromagnetic imaging (security and NDT) and localization, developing computational electromagnetics algorithms and technology on microwaves, millimeter wave and $\mathrm{THz}$ frequency bands. 